\title{
A BAYESIAN APPROACH FOR MODELING AND ANALYSIS OF CALL CENTER ARRIVALS
}

\author{
Xiaowei Zhang \\ Department of Industrial Engineering and Logistics Management \\ Hong Kong University of Science and Technology \\ Hong Kong, CHINA
}

\begin{abstract}
The Poisson process has been widely used in the literature to model call center arrivals. In recent years, however, there have been empirical studies suggesting the call arrival process has significant non-Poisson characteristics. In this paper, we introduce a new doubly stochastic Poisson model for call center arrivals and develop a Bayesian approach for the parameter estimation via the Markov chain Monte Carlo method. The model can well capture the call arrival process as illustrated by a case study.
\end{abstract}

\section{INTRODUCTION}

Telephone call centers, as the primary contact points between customers and their service providers, have become an integral part of today's economy. From the managerial perspective, to quantify the uncertainty of external call volume is essential for efficient staffing and scheduling of call center agents. To that end, an accurate and tractable model for call arrivals is necessary for evaluating system performance measures.

A widely adopted assumption in the literature of call center management is that calls arrive independently, so the call arrival process is typically modeled as a Poisson process; see Brown et al. (2005) for supportive hypothesis tests. However, the same article admits that the Poisson arrival rates are difficult to predict. Namely, there exists significant uncertainty in the arrival rates that ought to be treated carefully. Indeed, such an additional layer of randomness on top of the Poisson uncertainty makes both arrival forecasting and agent staffing challenging (see, for example, Gans et al. (2003)). Empirically, the uncertainty of the arrival rates is partially revealed by the burstiness of the call volume. Indeed, it has been observed in recent years that the call volume exhibits enormous overdispersion relative to the Poisson distribution; see, for example, Jongbloed and Koole (2001) and Avramidis et al. (2004). Both papers analyze a doubly stochastic Poisson model proposed in Whitt (1999), which characterizes the uncertainty of the arrival rates by a random variable, whose realized value indicates the "busyness" of the day. As noted in Avramidis et al. (2004), such a model results in an inaccurate correlation structure of the call volume, essentially because the randomness of the arrival rates is static.

In this paper, we present a dynamic doubly stochastic Poisson model that can characterize the uncertainty of the arrival rates much more accurately and develop its Bayesian analysis. Note that the Bayesian approach permits one to describe the uncertainty about the parameters of our model explicitly via specifying appropriate prior distributions. See also Soyer and Tarimcilar (2008) for a somewhat similar Bayesian approach for modeling call arrivals.

Other works on modeling call center arrivals include Bianchi et al. (1993), Andrews and Cunningham (1995), Weinberg et al. (2007), Shen and Huang (2008a), Shen and Huang (2008b), and Ibrahim and L'Ecuyer (2012). These papers all utilize time series models and succeed in forecasting intraday or interday call arrivals. Nevertheless, due to lack of domain knowledge, it might be difficult to derive managerial insights about the queueing dynamics from these models.

The rest of the paper is organized as follows. We introduce our model in Section 2 and conduct an extensive Bayesian analysis for our model in Section 3. We present a case study using real data to illustrate 
our model in Section 4. Section 5 gives concluding remarks including the potential extension of our model to incorporate other seasonal features such as the "day-of-week" effect. The proof of our main result is provided in the Appendix.

\section{DYNAMIC DOUBLY STOCHASTIC POISSON MODEL}

Several properties of call center arrivals must be taken into account when building a model. First, the arrival rate varies considerably with the time of day (i.e. the so-called "time-of-day" effect). Second, the call volume of a given time period typically has a much larger variance than that of a Poisson distribution (i.e. the overdispersion). Third, the call volumes of different time periods may be correlated. See Avramidis et al. (2004). Furthermore, one hopes the model to be parsimonious and analytically tractable.

Let $N(t)$ denote the number of calls that arrive during the time period $[0, t]$. We model the arrival process $(N(t): t \geq 0)$ as a doubly stochastic Poisson process with arrival rate process $(\lambda(t): t \geq 0)$. Namely, conditional on $\Lambda(t) \triangleq \int_{0}^{t} \lambda(s) \mathrm{d} t$, the distribution of $N(t)$ is Poisson given by

$$
\mathbb{P}(N(t)=n \mid \Lambda(t))=\frac{\Lambda(t)^{n} e^{-\Lambda(t)}}{n !} .
$$

It then follows that

$$
\mathbb{E}[N(t)]=\mathbb{E}[\mathbb{E}[N(t) \mid \Lambda(t)]]=\mathbb{E}[\Lambda(t)]
$$

SO

$$
\operatorname{Var}(N(t))=\operatorname{Var}[\mathbb{E}(N(t) \mid \Lambda(t))]+\mathbb{E}[\operatorname{Var}(N(t) \mid \Lambda(t))]=\operatorname{Var}(\Lambda(t))+\mathbb{E}[\Lambda(t)]>\mathbb{E}(N(t)),
$$

if $\Lambda(t)$ is random. Hence, doubly stochastic Poisson models can capture the overdispersion relative to the Poisson distribution.

Similarly as the model proposed in Whitt (1999), we use a multiplicative form for the arrival rate process, namely $\lambda(t)=\mu(t) g(t)$, where $\mu(t)$ is a positive deterministic function, capturing the "time-of-day" effect, and $G(t)$ is a stochastic process, governing the uncertainty of the arrival rate. We call our model as a dynamic doubly stochastic Poisson process because the uncertainty of the arrival rate in our model is time-evolving, whereas in the Whitt model, $g(t) \equiv G$ for some random variable $G$ so that the randomness of the arrival rate process is determined by $G$, thereby static. An implication of the static uncertainty is that if one observes the call center is unusually busy in the morning of a day, then one can predict for sure that the afternoon of the day will be busy as well. By contrast, our model permits a more flexible correlation structure of the call arrivals. Consequently, our model can well incorporate the three properties of call center arrivals aforementioned.

Although $g(t)$ could be virtually any arbitrary stochastic process to account for the overdispersion, one needs to consider several additional factors in order to build a reasonable model.

First of all, $g(t)$ should be positive and thus we consider an exponential form $g(x)=e^{x(t)}$.

Secondly, the model should be parsimonious. A continuous-time Markov chain model might be inappropriate because it is difficult to specify the number of states of the Markov chain and to estimate its transition probability matrix due to the large number of unknown parameters. Moreover, the model should be tractable. As a result, we decide to model $x(t)$ as an Ornstein-Uhlenbeck (OU) process because it is a low-parameter model with Gaussian transition densities.

In short, we model the arrival rate process as $\lambda(t)=\mu(t) e^{x(t)}$, where $\mu:[0, \infty) \rightarrow \mathbb{R}_{+}$is a positive deterministic function, and $x(t)$ is an OU process satisfying the following stochastic differential equation (SDE)

$$
\mathrm{d} x(t)=-\kappa x(t) \mathrm{d} t+\sigma \mathrm{d} B(t),
$$

where $\kappa$ and $\sigma$ are positive constants, and $(B(t): t \geq 0)$ is a standard Brownian motion.

Let $[0, T]$ denote the working hours of a working day for a call center and let $0=t_{0}<t_{1}<\cdots<t_{k}=T$ be a partition of $[0, T]$. In practice, the partition depends on factors such as data availability or agent 
scheduling considerations. Assume $\mu(\cdot)$ is a piecewise constant function defined below

$$
\mu(t)=\theta_{i}, \quad \text { if } t \in\left[t_{i-1}, t_{i}\right)
$$

where $\theta_{i}$ is a positive constant for $i=1, \ldots, k$. Further, assume $\mu(t)$ is periodic with period $T$.

In what follows, we will present a Bayesian analysis of our model. The Bayesian approach requires that uncertainty about all unknown parameters, including $\kappa, \sigma$, and $\Theta=\left(\theta_{i}: i=1, \ldots, k\right)$, is characterized probabilistically via a prior (joint) distribution of $(\kappa, \sigma, \Theta)$. Note that there are two major difficulties in estimating the unknown parameters. First, the likelihood function of the observations of the arrival process $N(t)$ has no analytical expression and its calculation requires "integrating out" the unobservable arrival rate process, which is a prohibitively high dimensional integration problem. Second, the number of unknown parameters is large. For instance, if $[0, T]$ is partitioned half-hourly, which is typically assumed in practice when modeling the arrival process as an inhomogeneous Poisson process, there would be more than 30 unknown parameters in total. Hence, the numerical optimization problem associated with any maximum likelihood estimation method would be overwhelmingly difficult. The Bayesian treatment provides an alternate (and possibly the only) feasible approach for estimating the unknown parameters.

\section{BAYESIAN ANALYSIS OF THE MODEL}

Assume that the arrival process $N(\cdot)$ is observed at equally spaced time epochs $\{i \delta: i=0,1, \ldots,(m+1) \delta\}$, but the analysis can be extended to any sequence of time epochs without essential difficulty except for notational complexity. To simplify notations, we let $X_{i}=x(i \delta), Y_{i}=N((i+1) \delta)-N(i \delta)$, so that $\mathbf{X}=\left\{X_{0}, X_{1}, \ldots, X_{m}\right\}$ is the vector of unobserved arrival rates at observation time epochs and $\mathbf{Y}=\left\{Y_{0}, Y_{1}, \ldots, Y_{m}\right\}$ is the observed data.

Let $\Xi$ denote all the unknown parameters, i.e. $\Xi=(\kappa, \sigma, \Theta)$. The central piece of the Bayesian analysis is to compute $p(\Xi, \mathbf{X} \mid \mathbf{Y})$, the posterior joint distribution of the unknown parameters and the unobserved arrival rates conditional on the observations. From the computational perspective, it is prohibitively involved to generate samples of $(\Xi, \mathbf{X})$ from this enormously high dimensional conditional distribution due to lack of an analytically tractable form. Nonetheless, the Markov chain Monte Carlo (MCMC) method accompanied by the Gibbs sampler provides a feasible solution to explore this distribution via simulating a Markov chain whose stationary distribution is $p(\Xi, \mathbf{X} \mid \mathbf{Y})$. We refer to Robert and Casella (2005) for an extensive treatment of the MCMC method and refer to Gelfand and Smith (1990) for a review of the Gibbs sampler.

The Gibbs sampler permits to break down the posterior distribution of $(\Xi, \mathbf{X})$ into a group of onedimensional conditional distributions. In particular, the Hammersley-Clifford theorem (see Hammersley and Clifford 1970) implies that $p(\Xi, \mathbf{X} \mid \mathbf{Y})$ is uniquely determined by its full conditionals, including

$$
\begin{aligned}
& p\left(X_{i} \mid \mathbf{X}_{-i}, \mathbf{Y}, \Xi\right), \quad i=0,1, \ldots, m, \\
& p(\kappa \mid \sigma, \Theta, \mathbf{X}, \mathbf{Y}), \\
& p(\sigma \mid \kappa, \Theta, \mathbf{X}, \mathbf{Y}), \text { and } \\
& p\left(\theta_{i} \mid \kappa, \sigma, \Theta_{-i}, \mathbf{X}, \mathbf{Y}\right), \quad i=1, \ldots, k
\end{aligned}
$$

where $\Theta_{-i}=\left\{\theta_{j}: j \neq i\right\}$ and $\mathbf{X}_{-i}=\left\{X_{j}: j \neq i\right\}$.

In order to compute the above full conditionals to analytically tractable form, we adopt the following two approximation. First, we approximate the one-step transition distribution of the Markov chain $\left\{X_{0}, X_{1}, \ldots, X_{m}\right\}$ via the Euler discretization scheme (see, for example, Amussen and Glynn 2007) of the SDE (1), i.e.

$$
X_{j+1}-X_{j} \approx-\kappa X_{j} \delta+\sigma \sqrt{\delta} \varepsilon_{j+1},
$$

where $\varepsilon_{i}$ 's are i.i.d. standard normal random variables. So

$$
p\left(X_{j+1} \mid X_{j}, \Xi\right) \approx \frac{1}{\sqrt{2 \delta \sigma^{2}}} \exp \left(-\frac{\left(X_{j+1}-(1-\kappa \delta) X_{j}\right)^{2}}{2 \delta \sigma^{2}}\right) .
$$


Second, given $\mathbf{X}$ and $\Xi$, we approximately treat $Y_{j}$ as a Poisson random variable with mean

$$
\int_{j \delta}^{(j+1) \delta} \mu(s) e^{x(s)} \mathrm{d} s \approx \theta_{I(j)} \delta e^{X_{j}}
$$

where $I(j)=i$ if $\mu(j \delta)=\theta_{i}$, and treat $\left\{Y_{0}, Y_{1}, \ldots, Y_{m}\right\}$ as conditional independent. Hence,

$$
p(\mathbf{Y} \mid \mathbf{X}, \Xi) \approx \prod_{j=0}^{m} p\left(Y_{j} \mid X_{j}, \Xi\right) \approx \prod_{j=0}^{m} \frac{\left(\theta_{I(j)} \delta e^{X_{j}}\right)^{Y_{j}}}{\left(Y_{j}\right) !} \exp \left(-\theta_{I(j)} \delta e^{X_{j}}\right) .
$$

Note that for a typical call center, the observation resolution parameter $\delta$ can be very small, e.g. $\delta<1 / 10$ hour, so the approximations (7) and (8) can be very accurate.

In light of (7) and (8), the full conditionals (3) - (6) can now be reduced to analytically tractable forms. Note that

$$
\begin{aligned}
p\left(X_{i} \mid \mathbf{X}_{-i}, \mathbf{Y}, \Xi\right) & \propto p(\mathbf{X}, \Xi) \cdot p(\mathbf{Y} \mid \mathbf{X}, \Xi) \\
& =p\left(X_{i} \mid \mathbf{X}_{-i}, \Xi\right) \cdot p\left(\mathbf{X}_{-i}, \Xi\right) \cdot \prod_{j=0}^{m} p\left(Y_{j} \mid X_{j}, \Xi\right) \\
& \propto p\left(X_{i} \mid \mathbf{X}_{-i}, \Xi\right) \cdot p\left(Y_{i} \mid X_{i}, \Xi\right),
\end{aligned}
$$

where the last equality holds because any item not involving $X_{i}$ can be viewed as constant. Moreover, the Markov property of $\mathbf{X}$ implies that for $i=1, \ldots, m-1$,

$$
p\left(X_{i} \mid \mathbf{X}_{-i}, \Xi\right)=p\left(X_{i} \mid X_{i-1}, X_{i+1}, \Xi\right) \propto p\left(X_{i} \mid X_{i-1}, \Xi\right) \cdot p\left(X_{i+1} \mid X_{i}, \Xi\right) .
$$

It then follows from (9) and (10) that

$$
p\left(X_{i} \mid \mathbf{X}_{-i}, \mathbf{Y}, \Xi\right) \propto p\left(X_{i} \mid X_{i-1}, \Xi\right) \cdot p\left(X_{i+1} \mid X_{i}, \Xi\right) \cdot p\left(Y_{i} \mid X_{i}, \Xi\right),
$$

for $i=1, \ldots, m-1$. At the two end points, we have the obvious corrections by setting $p\left(X_{i} \mid X_{i-1}, \Xi\right)=1$ for $i=1$ and setting $p\left(X_{i+1} \mid X_{i}, \Xi\right)=1$ for $i=m$. Since the right-hand-side of (11) can be computed analytically by (7) and (8), the Metropolis-Hastings algorithm can easily be applied to generate samples from the conditional distribution $p\left(X_{i} \mid \mathbf{X}_{-i}, \mathbf{Y}, \Xi\right)$; see, for example, Robert and Casella (2005).

On the other hand, conjugate prior distributions for $\Xi$ can be found for the posterior distributions (4), (5), and (6). In particular, we have the following theorem, whose proof is given in Appendix.

Theorem 1 Suppose that the approximations (7) and (8) are applied. Assume that the priors of $\Xi$ are given by

$$
\begin{aligned}
& \kappa \sim \text { Gaussian, } \\
& \sigma^{2} \sim \text { inverse Gamma, and } \\
& \theta_{i} \sim \text { Gamma }, \quad i=1, \ldots, k
\end{aligned}
$$

and that the above priors are mutually independent. Then,

$$
\begin{aligned}
& \kappa \mid \sigma, \Theta, \mathbf{X}, \mathbf{Y} \sim \text { Gaussian, } \\
& \sigma^{2} \mid \kappa, \Theta, \mathbf{X}, \mathbf{Y} \sim \text { inverse Gamma, and } \\
& \theta_{i} \mid \kappa, \sigma, \Theta_{-i}, \mathbf{X}, \mathbf{Y} \sim \text { Gamma }, \quad i=1, \ldots, k
\end{aligned}
$$


By virtue of Theorem 1, we can implement the Gibbs sampler to $\left\{\left(\Xi^{(j)}, \mathbf{X}^{(j)}\right): j=0, \ldots, J\right\}$, where $J$ is the number of samples one hopes to generate, from the posterior distribution $p(\Xi, \mathbf{X} \mid \mathbf{Y})$ as follows.

1. Initialize an estimate of $(\Xi, \mathbf{X})$ at $\left(\Xi^{(0)}, \mathbf{X}^{(0)}\right)$.

2. Given $\left(\Xi^{(j)}, \mathbf{X}^{(j)}\right)$,

(a) simulate $\kappa^{(j+1)}$ from the Gaussian posterior $p\left(\kappa \mid \sigma^{(j)}, \Theta^{(j)}, \mathbf{X}^{(j)}, \mathbf{Y}\right)$ by (13);

(b) simulate $\left(\sigma^{2}\right)^{(j+1)}$ from the inverse Gamma posterior $p\left(\sigma^{2} \mid \kappa^{(j)}, \Theta^{(j)}, \mathbf{X}^{(j)}, \mathbf{Y}\right)$ by (14);

(c) simulate $\theta_{i}^{(j+1)}$ from the Gamma posterior $p\left(\theta_{i} \mid \kappa^{(j)}, \sigma^{(j)}, \Theta_{-i}^{(j)}, \mathbf{X}^{(j)}, \mathbf{Y}\right)$ for $i=1, \ldots, k$ by (15);

(d) simulate $X_{i}^{(j+1)}$ from the posterior $p\left(X_{i} \mid \mathbf{X}_{-i}^{(j)}, \Xi^{(j)}, \mathbf{Y}\right)$ by (11) with the Metropolis-Hastings algorithm, for $i=0,1, \ldots, m$.

3. Return to Step 2 until the desired number of samples have been simulated.

Note that the posterior predictive distribution of the arrival counts in $[s, t]$ is Poisson with mean

$$
\mathbb{E}\left(\int_{s}^{t} \mu(r) e^{x(r)} \mathrm{d} r \mid \mathbf{Y}\right) \approx \mathbb{E}\left(\sum_{l=r_{1}}^{r_{2}-1} \delta \mu(l \delta) e^{X_{l}} \mid \Xi, \mathbf{X}\right) \mathrm{d} p(\Xi, \mathbf{X} \mid \mathbf{Y}),
$$

assuming $s=r_{1} \delta$ and $t=r_{2} \delta$. Hence, we can assess the goodness-of-fit by evaluating the error between the observed arrival counts and the posterior mean of the arrival counts (12), which can be approximated by the posterior sample mean

$$
\frac{1}{J} \sum_{j=1}^{J} \sum_{l=r_{1}}^{r_{2}-1} \delta \mu^{(j)}(l \delta) e^{X_{l}^{(j)}}
$$

\section{A CASE STUDY}

In this section, we apply our model to a real call center case. The data set used here is publicly available at the Service Enterprise Engineering (SEE) Center, Technion. It is from an anonymous call center in Israel and contains all the telephone records in November and December 1999. We analyze only the weekdays, because the call arrivals on weekends obviously have a distinctive pattern and require a slightly revised parameter specification. As will be seen later we easily can extend our model to reflect the "day-of-week" effect when necessary.

The call center opens at $7 \mathrm{am}$ and closes at midnight on weekdays and there are 44 weekdays in the data set. For simplicity, we assume that the call center operates "nonstoppingly" in the sense that each weekday has 17 hours and there are no weekends. Further, we divide each day into 34 half-hourly subintervals, so the time-of-day factor $\mu(t)$ defined in (2) takes values from $\Theta=\left\{\theta_{i}: i=0,1, \ldots, 33\right\}$. We plot average arrival count in each of the 34 subintervals across the 44 weekdays as well as the $95 \%$ band of the observations in Figure 1. Note that under the Poisson model the arrival count has a Poisson distribution and thus its variance equals its mean. We also plot the $95 \%$ confidence intervals under the Poisson model in Figure 1. Clearly, the arrival counts have a much larger variance than what the Poisson model suggests, which demonstrates a fundamental flaw of the Poisson model.

When running the Bayesian analysis, we draw 20,000 MCMC samples and discard the first half. We use the trace plots of the unknown parameters to ensure that the Gibbs sampler has approximately converged and that it does not depend significantly on its initial value. See Chapter 11.6 of Gelman et al. (2004) for a further discussion on the convergence assessment of the MCMC method. In Figure 2, we plot the time-of-day factor $\mu(t)$ (or equivalently, $\theta_{i}$ 's) as well as its associated $95 \%$ posterior confidence band based on the 10,000 effective MCMC samples and compare it with the mean arrival numbers in each half-hourly subintervals. Obviously, $\mu(t)$ well captures the time-of-day effect.

Moreover, the histograms of $\kappa, \sigma$, and several selected $\theta_{i}$ 's are shown in Figure 3. The mean and standard deviation of each unknown parameter are reported in Table 1. 


\section{Zhang}

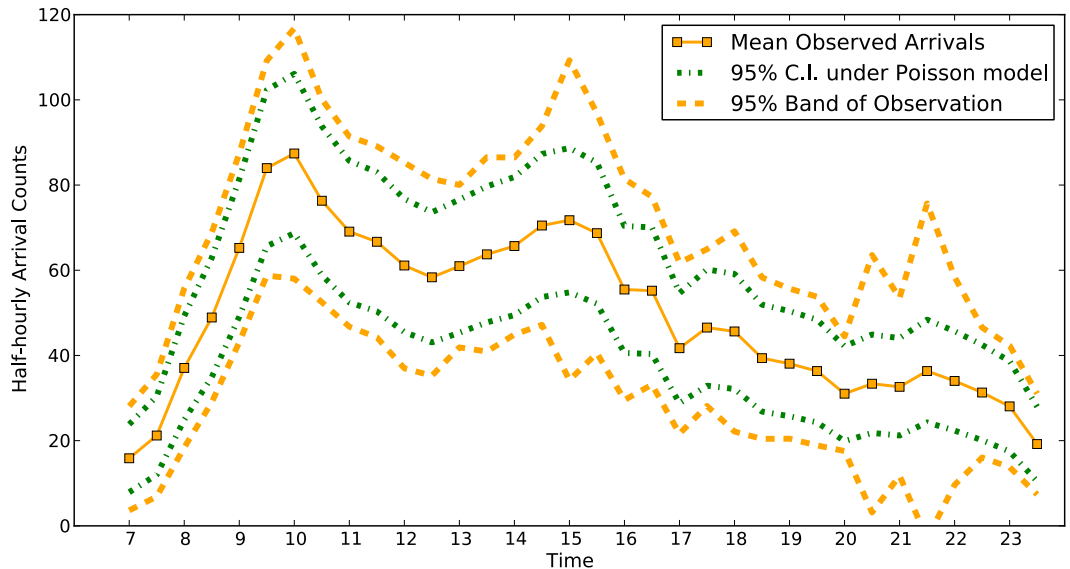

Figure 1: Time-of-day effect and overdispersion relative to the Poisson distribution.

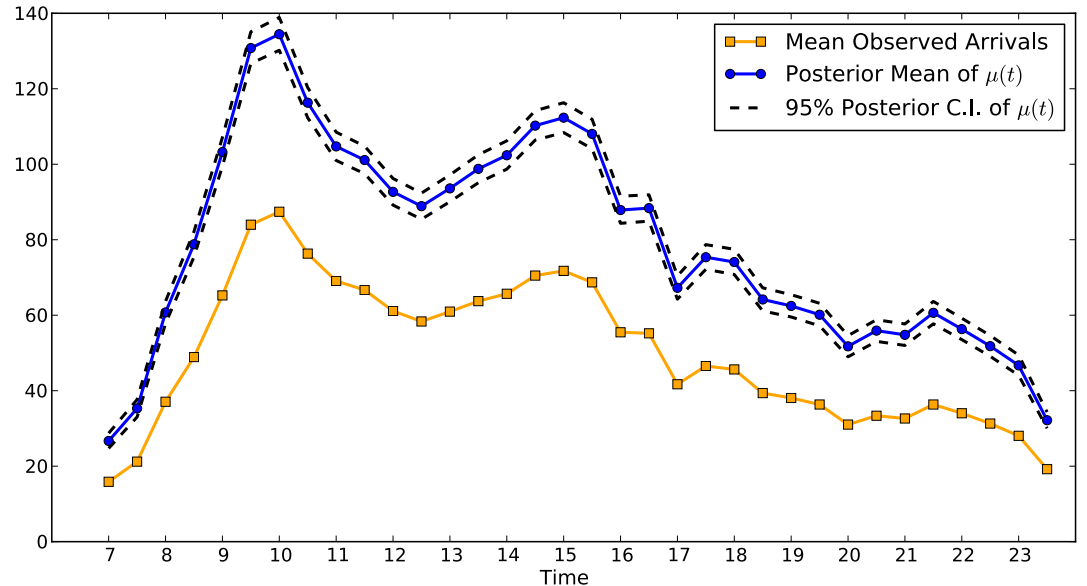

Figure 2: Time-of-day factor $\mu(t)$ and mean arrival counts.

Table 1: Estimation results of the unknown parameters.

\begin{tabular}{c|c|c|c|c|c|c|c|c|c}
\hline & $\kappa$ & $\sigma$ & $\theta_{0}$ & $\theta_{1}$ & $\theta_{2}$ & $\theta_{3}$ & $\theta_{4}$ & $\theta_{5}$ & $\theta_{6}$ \\
\hline Mean & $1.55 \mathrm{E}-02$ & $4.05 \mathrm{E}-02$ & 26.68 & 35.31 & 60.73 & 78.81 & 103.23 & 130.78 & 134.46 \\
\hline S.D. & $5.98 \mathrm{E}-03$ & $3.35 \mathrm{E}-04$ & 1.02 & 1.18 & 1.51 & 1.71 & 1.94 & 2.16 & 2.18 \\
\hline \hline & $\theta_{7}$ & $\theta_{8}$ & $\theta_{9}$ & $\theta_{10}$ & $\theta_{11}$ & $\theta_{12}$ & $\theta_{13}$ & $\theta_{14}$ & $\theta_{15}$ \\
\hline Mean & 116.31 & 104.72 & 101.11 & 92.69 & 88.89 & 93.60 & 98.78 & 102.38 & 110.23 \\
\hline S.D. & 2.06 & 1.91 & 1.88 & 1.80 & 1.76 & 1.82 & 1.89 & 1.91 & 2.02 \\
\hline \hline & $\theta_{16}$ & $\theta_{17}$ & $\theta_{18}$ & $\theta_{19}$ & $\theta_{20}$ & $\theta_{21}$ & $\theta_{22}$ & $\theta_{23}$ & $\theta_{24}$ \\
\hline Mean & 112.33 & 108.04 & 87.87 & 88.38 & 67.26 & 75.37 & 74.09 & 64.17 & 62.46 \\
\hline S.D. & 2.03 & 2.00 & 1.82 & 1.79 & 1.58 & 1.67 & 1.69 & 1.57 & 1.52 \\
\hline \hline & $\theta_{25}$ & $\theta_{26}$ & $\theta_{27}$ & $\theta_{28}$ & $\theta_{29}$ & $\theta_{30}$ & $\theta_{31}$ & $\theta_{32}$ & $\theta_{33}$ \\
\hline Mean & 60.13 & 51.75 & 55.92 & 54.80 & 60.64 & 56.32 & 51.81 & 46.71 & 32.17 \\
\hline S.D. & 1.51 & 1.41 & 1.46 & 1.46 & 1.52 & 1.45 & 1.41 & 1.35 & 1.10 \\
\hline
\end{tabular}




\section{Zhang}
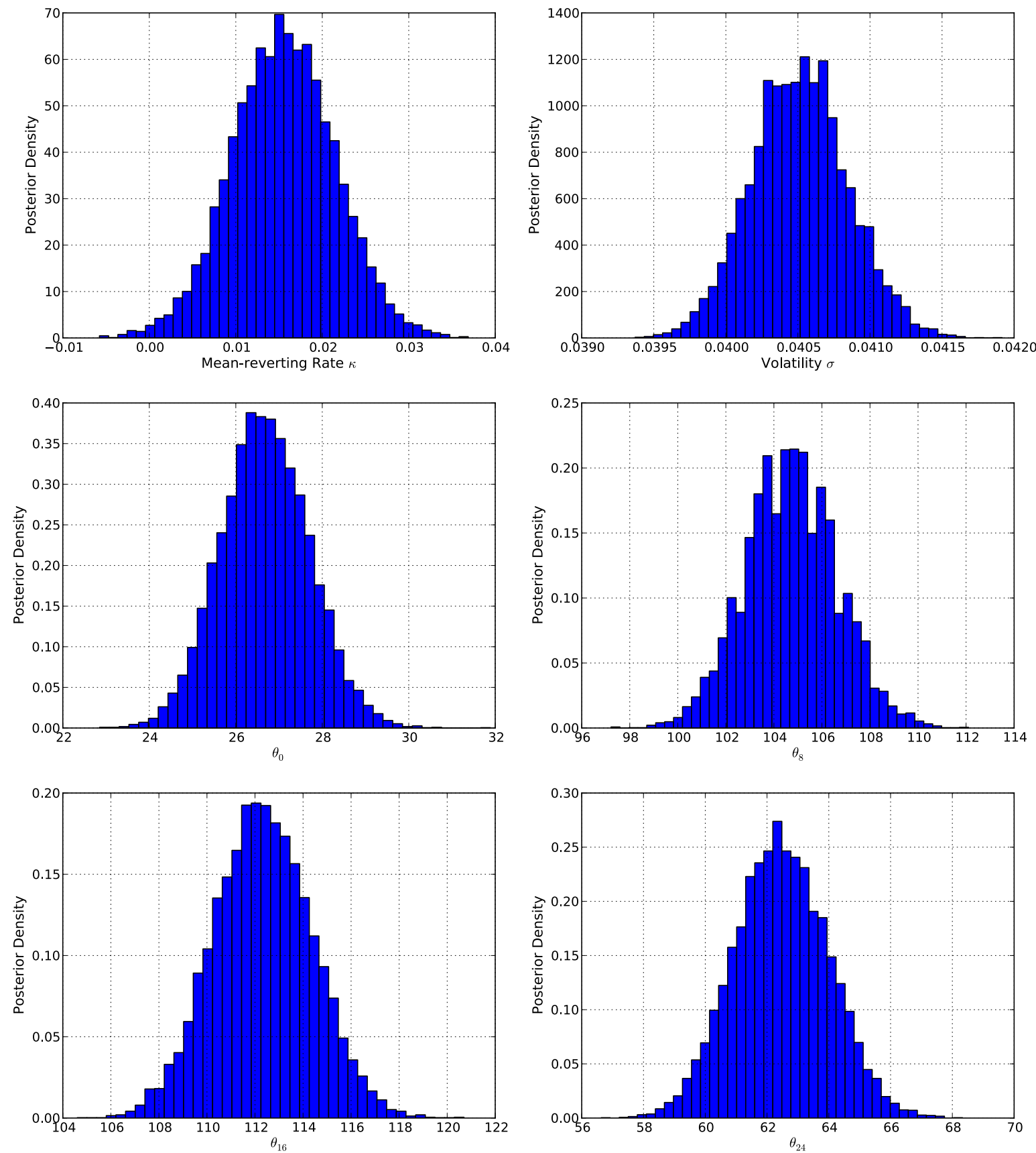

Figure 3: Histograms of some unknown parameters. 


\section{Zhang}
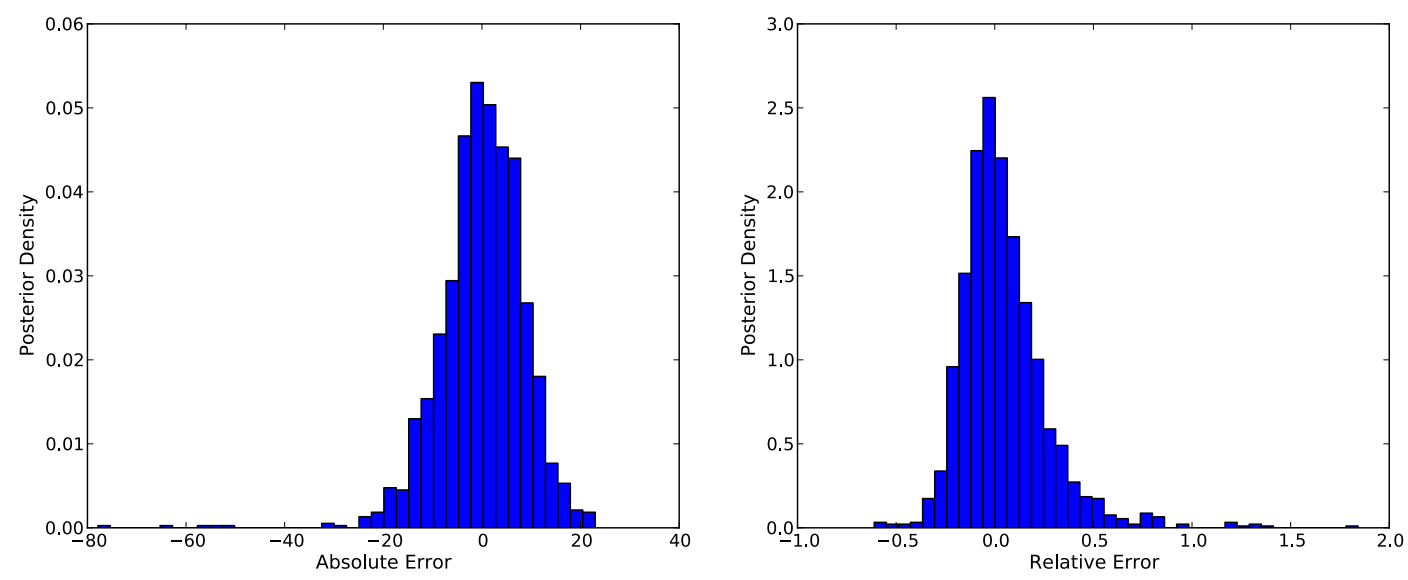

Figure 4: Histograms of the absolute error and the relative error of the posterior prediction.

Finally, we assess the goodness-of-fit by comparing the observed arrival counts and the posterior mean of the arrival counts in each of the $34 \times 44=1,496$ half-hourly subintervals. The histogram of the absolute errors and the relative errors are shown in Figure 4. Moreover, in Figure 5 we plot the posterior mean of the arrival counts in the half-hourly subintervals of the first five weekdays as well as their associated 95\% confidence intervals and compare them with the 95\% confidence intervals under the Poisson model. Obviously, our model significantly reduces the estimation variance by introducing only two additional parameter $\kappa$ and $\sigma$. The fitting for the other weekdays is similarly well.

\section{CONCLUDING REMARKS}

In this paper, we have introduced a doubly stochastic Poisson model for the call arrival process of a call center. The arrival rate has a multiplicative structure which consists of a deterministic part and a stochastic part. The former captures the "time-of-day" effect whereas the latter governs the uncertainty of the arrival rate. We have also developed a tractable Bayesian analysis in which the unknown parameters can be simulated via conjugate priors whereas the latent variables via the Gibbs sampler. Finally, we have presented a real case study which demonstrates our model can fit the data very well and it is significantly superior to the widely used Poisson model, especially considering our model has only two more parameters $\kappa$ and $\sigma$.

If one wants to incorporate the weekends or the "day-of-week" effect is apparent, then one can revise the model such that each weekday/weekend has a set of parameters $(\kappa, \sigma, \Theta)$. The Bayesian analysis derived in this paper then can be applied after a simple modification.

In practice, one might not be able to a priori identify the appropriate specification of the parameters, which calls for a model selection procedure. One can either estimate the Bayes factors (see Kass and Raftery 1995) or other criteria such as the Bayesian information criterion (BIC) and the deviance information criterion (DIC) (see Gelman et al. (2004)). 


\section{Zhang}

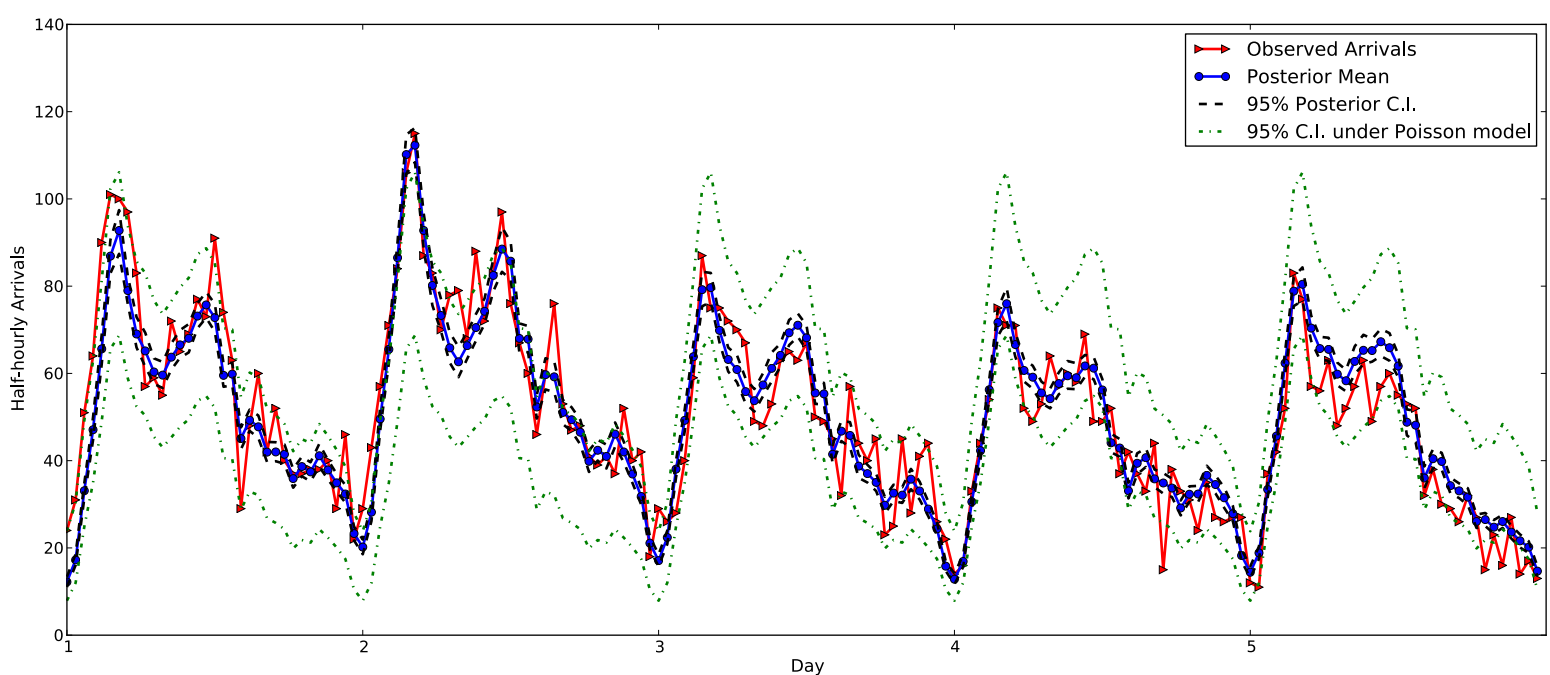

Figure 5: The $95 \%$ posterior confidence intervals of the arrival counts under our model, the $95 \%$ confidence intervals of the arrival counts under the Poisson model, and the observed arrival counts in the half-hourly subintervals of the first five weekdays.

\section{A APPENDICES}

Proof of Theorem 1 First of all, suppose that the prior distribution of $\kappa$ is Gaussian with mean $a_{1}$ and standard deviation $b_{1}$. Noting that $p(\mathbf{Y} \mid \mathbf{X}, \Xi)$ does not involve $\kappa$ by (8) and applying (7),

$$
\begin{aligned}
p(\kappa \mid \sigma, \Theta, \mathbf{X}, \mathbf{Y}) & \propto p(\Xi, \mathbf{X}, \mathbf{Y})=p(\mathbf{Y} \mid \mathbf{X}, \Xi) \cdot p(\mathbf{X} \mid \Xi) \cdot p(\Xi) \\
& \propto \prod_{j=0}^{m-1} p\left(X_{i+1} \mid X_{i}, \Xi\right) \cdot p(\kappa), \\
& =\prod_{j=0}^{m-1}\left[\frac{1}{\sqrt{2 \pi \delta \sigma^{2}}} \exp \left(-\frac{\sum_{j=0}^{m-1}\left(X_{j+1}-(1-\kappa \delta) X_{j}\right)^{2}}{2 \delta \sigma^{2}}\right)\right] \cdot \frac{1}{\sqrt{2 \pi b_{1}^{2}}} \exp \left(-\frac{\left(\kappa-a_{1}\right)^{2}}{2 b_{1}^{2}}\right) \\
& \propto \exp \left[-\kappa^{2}\left(\frac{1}{2 b_{1}^{2}}+\sum_{j=0}^{m-1} \frac{\delta X_{j}^{2}}{2 \sigma^{2}}\right)+\kappa\left(\frac{a_{1}}{b_{1}^{2}}-\sum_{j=0}^{m-1} \frac{X_{j}\left(X_{j+1}-X_{j}\right)}{\sigma^{2}}\right)\right] .
\end{aligned}
$$

Hence, $\kappa \mid \sigma, \Theta, \mathbf{X}, \mathbf{Y}$ is Gaussian with mean $\frac{B}{A}$ and variance $\frac{1}{A}$, where

$$
A=\frac{1}{b_{1}^{2}}+\frac{\delta}{\sigma^{2}} \sum_{j=0}^{m-1} X_{j}^{2} \quad \text { and } \quad B=\frac{a_{1}}{b_{1}^{2}}-\frac{1}{\sigma^{2}} \sum_{j=0}^{m-1}\left(X_{j}\left(X_{j+1}-X_{j}\right)\right) .
$$


Similarly, supposing that the prior distribution of $\sigma^{2}$ is inverse Gamma with shape parameter $a_{2}$ and scale parameter $b_{2}$, we have

$$
\begin{aligned}
p\left(\sigma^{2} \mid \kappa, \Theta, \mathbf{X}, \mathbf{Y}\right) & \propto \prod_{j=0}^{m-1} p\left(X_{i+1} \mid X_{i}, \Xi\right) \cdot p\left(\sigma^{2}\right) \\
& =\prod_{j=0}^{m-1}\left[\frac{1}{\sqrt{2 \pi \delta \sigma^{2}}} \exp \left(-\frac{\left(X_{j+1}-(1-\kappa \delta) X_{j}\right)^{2}}{2 \delta \sigma^{2}}\right)\right] \cdot \frac{b_{2}^{a_{2}}}{\Gamma\left(a_{2}\right)}\left(\sigma^{2}\right)^{-a_{2}-1} \exp \left(\frac{-b_{2}}{\sigma^{2}}\right) \\
& \propto\left(\sigma^{2}\right)^{-a_{2}-1-\frac{m}{2}} \exp \left[\frac{1}{\sigma^{2}}\left(-b_{2}-\sum_{j=0}^{m-1}\left(X_{j+1}-(1-\kappa \delta) X_{j}\right)^{2}\right)\right]
\end{aligned}
$$

implying that $\sigma^{2} \mid \kappa, \Theta, \mathbf{X}, \mathbf{Y}$ is inverse Gamma with shape parameter $a_{2}+\frac{m}{2}$ and scale parameter

$$
b_{2}+\sum_{j=0}^{m-1}\left(X_{j+1}-(1-\kappa \delta) X_{j}\right)^{2} .
$$

Lastly, suppose that the prior distribution of $\theta_{i}$ is Gamma with shape parameter $c_{i}$ and rate parameter $d_{i}$. Then, noting that $p(\mathbf{X} \mid \Xi)$ does not involve $\Theta$ by (7) and applying (8),

$$
\begin{aligned}
p\left(\theta_{i} \mid \kappa, \sigma, \Theta_{-i}, \mathbf{X}, \mathbf{Y}\right) & \propto p(\Xi, \mathbf{X}, \mathbf{Y})=p(\mathbf{Y} \mid \mathbf{X}, \Xi) \cdot p(\mathbf{X} \mid \Xi) \cdot p(\Xi) \\
& \propto \prod_{\left\{j: \mu(j \delta)=\theta_{i}\right\}} p\left(Y_{j} \mid X_{j}, \Xi\right) \cdot p\left(\theta_{i}\right) \\
& =\prod_{\left\{j: \mu(j \delta)=\theta_{i}\right\}} \frac{\left(\theta_{i} \delta e^{X_{j}}\right)^{Y_{j}}}{\left(Y_{j}\right) !} \exp \left(-\theta_{i} \delta e^{X_{j}}\right) \cdot \frac{1}{\Gamma\left(c_{i}\right) d_{i}^{c_{i}}} \theta_{i}^{c_{i}-1} \exp \left(-d_{i} \theta_{i}\right) \\
& \propto \theta_{i}^{c_{i}-1+\sum_{j} Y_{j}} \exp \left[-\theta_{i}\left(d_{i}+\delta \sum_{j} e^{X_{j}}\right)\right] .
\end{aligned}
$$

Therefore, $\theta_{i} \mid \kappa, \sigma, \Theta_{-i}, \mathbf{X}, \mathbf{Y}$ is Gamma with shape parameter $C_{i}$ and rate parameter $D_{i}$, where

$$
C_{i}=c_{i}+\sum_{\left\{j: \mu(j \delta)=\theta_{i}\right\}} Y_{j} \quad \text { and } \quad D_{i}=d_{i}+\delta \sum_{\left\{j: \mu(j \delta)=\theta_{i}\right\}} e^{X_{j}}
$$

\section{REFERENCES}

Amussen, S., and P. W. Glynn. 2007. Stochastic Simulation: Algorithm and Analysis. Springer-Verlag.

Andrews, B. H., and S. M. Cunningham. 1995. "L. L. Bean Improves Call-center Forecasting”. Interfaces 25 (6): $1-13$.

Avramidis, A. N., A. Deslauriers, and P. L'Ecuyer. 2004. "Modeling Daily Arrivals to a Telephone Call Center". Management Science 50 (7): 896-908.

Bianchi, L., J. E. Jarrett, and R. C. Hanumara. 1993. "Forecasting Incoming Calls to Telemarketing Centers". Journal of Business Forecasting Methods and Systems 12:3-12.

Brown, L., N. Gans, A. Mandelbaum, A. Sakov, H. Shen, S. Zeltyn, and L. Zhao. 2005. "Statistical Analysis of a Telephone Call Center: A Queueing-science Perspective”. J. Amer. Statist. Assoc. 100 (1): 36-50.

Gans, N., G. Koole, and A. Mandelbaum. 2003. "Telephone Call Centers: Tutorial, Review, and Research Prospects". Manufacturing \& Service Operations Management 5 (2): 79-141.

Gelfand, A. E., and A. F. M. Smith. 1990. "Sampling-based Approaches to Calculating Marginal Densities". J. Amer. Statist. Assoc. 85 (410): 398-409.

Gelman, A., J. B. Carlin, H. S. Stern, and D. B. Rubin. 2004. Bayesian Data Analysis. 2nd ed. Chapman and Hall/CRC. 
Hammersley, J. M., and P. Clifford. 1970. "Markov Fields on Finite Graphs and Lattices". unpublished. Ibrahim, R., and P. L'Ecuyer. 2012. "Forecasting Call Center Arrivals: Fixed-effects, Mixed-effects, and Bivariate Models". Manufacturing \& Service Operations Management, forthcoming.

Jongbloed, G., and G. Koole. 2001. "Managing Uncertainty in Call Centers Using Poisson Mixtures". Appl. Stochastic Models Bus. Indust. 17:307-318.

Kass, R. E., and A. E. Raftery. 1995. "Bayesian Factors". J. Amer. Statist. Assoc. 90 (430): 773-795.

Robert, C., and G. Casella. 2005. Monte Carlo Statistical Methods. 2nd ed. Springer.

Shen, H., and J. Huang. 2008a. "Forecasting Time Series of Inhomogeneous Poisson Process with Application to Call Center Workforce Management". Annals of Applied Statistics 2:601-623.

Shen, H., and J. Huang. 2008b. "Intraday Forecasting and Interday Updating of Call Center Arrivals". Manufacturing \& Service Operations Management 10:391-410.

Soyer, R., and M. M. Tarimcilar. 2008. "Modeling and Analysis of Call Center Arrival Data: A Bayesian Approach”. Management Science 54 (2): 266-278.

Weinberg, J., L. D. Brown, and J. R. Stroud. 2007. "Bayesian Forecasting of an Inhomogeneous Poisson Process with Applications to Call Center Data”. J. Amer. Statist. Assoc. 102:1185-1198.

Whitt, W. 1999. "Dynamic Staffing in a Telephone Call Center Aiming to Immediately Answer All Calls". Oper. Res. Lett. 24:205-212.

\section{AUTHOR BIOGRAPHIES}

XIAOWEI ZHANG is an Assistant Professor in the Department of Industrial Engineering and Logistics Management at Hong Kong University of Science and Technology. He received his $\mathrm{Ph} . \mathrm{D}$. in Operations Research from Stanford University in 2011. He is a member of INFORMS and his research interests include rare event simulation and stochastic modeling in service engineering. His email address is xiaoweiz@ust.hk. 